\title{
Representing and selecting problems through contradictions clouds
}

\author{
Denis Cavallucci ${ }^{1}$, François Rousselot ${ }^{2}$, and Cécilia Zanni $^{3}$ \\ 1,2\&3 Design Engineering Laboratory (LGeCo), National Instituted of Applied \\ Sciences (INSA) Strasbourg. FRANCE, \{denis.cavallucci, francois.rousselot, \\ cecilia.zanni\}@insa-strasbourg.fr
}

\begin{abstract}
Within the scope of Computer Aided Innovation, it is commonly agreed that problem formulation remains in need of robust improvement. Prior to this, means of conducting and representing problems are needed, so as the way a software implementation would help designers, for instance, in decision making. Our approach presents the originality of including the use of a contradiction formalism associated to the notions of problem network, parameter network and their interactions. Several other research outcomes have clearly presented interesting results in the direction of problem network constitution and defined its role within problem formulation and inventive design but have not yet clarified the way these networks are linked together. In this article, we present the notion of contradiction clouds and its manipulation in order to fulfil the objective of assisting decisions in a more advanced way than an intuitive decision method. These proposed rules of representation are based on the assumption that contradictions need to be associated with means of characterization for a graphical representation to be relevant and a decision to be engaged. A test procedure of our proposals has been conducted in two industrial case studies; one in a steel maker company and a second in an automotive supplier.
\end{abstract}

Keywords: TRIZ, Contradictions clouds, Inventive Design, R\&D decisions

\section{Introduction}

\subsection{The place of invention in innovation}

It is now acknowledged in scientific and industrial circles that there is a major difference between innovation and invention [1]. Many works have highlighted these differences but can be summarized in the fields of engineering as follows:

Please use the following format when citing this chapter: 
The status of Innovation (associated to the object or more largely what man have built: artifacts) is acquired whenever a society adoption of this same artifact appears as new in a group of consumers having a certain legitimacy.

The question then arouses about the role of invention in this scheme. Invention, being the cognitive fruit of inventors materialized in an innovative technical solution associated with its resultant in the novel object, this statement is not obviously connected with the concept of innovation described above. It is thus interesting to investigate which new facts can be at the origin of invention and in which ways they can result in an impact on innovation.

In other words, an innovation can have nothing inventive in it, just like an invention may never result in an innovation. The only semantic parallel between these two terms lies in the novelty of the idea for one, of the society adoption for the other. Moreover, the temporal aspect of states (both invention and innovation) for the same object remains often timely distant. Sometimes, as it was the case for photography, one century occurs before an invention does acquire the state of innovation. This rhythm seems to accelerate as the decades our industries cross, moreover since the era of innovation became a reality. Therefore the rhythm of emergence of inventions is ever more solicited with the necessity for a company to ever be intensively proposing innovations.

This distant temporal aspect between invention and innovation can nevertheless inspire a reflection: "does the original presence of an invention support the emergence of an innovation?" or "is this presence guaranteeing a particular kind of innovation, such as for example provoking a broader success of a more durable notoriety?"

By observing innovations which have landmarked this century, a brief reply is undoubtedly in favor of this statement, innovations which landmarked this century have in their immense majority at their origin a relevant invention and of a high inventive value (within the meaning of Altshuller's levels of inventiveness). We thus question also on the objective methods which characterize the emergence of inventions, since they seem to be sources of innovations provoking successes for companies.

\subsection{Which fundamentals make a mode of design "inventively- driven?"}

In this section, we will start by clarifying the confused relation between creativity, often perceived and considered from the angle of a process or a stage of this process, and an inventive behavior which results rather in a particular capacity to address problem solving having certain specificities.

Creativity, seeming more and more a recognized process of a more global activity of design, is at the heart of many reflections [2]. We can draw this statement just by observing the ever increasing contributions these last years in the fields of Design Sciences around creative phases which tries to explain that time 
has come to increase in their effectiveness [3]. Let us add to that the emergence of theoretical and practical contributions to characterize them, like those around TRIZ. They confirm the tendency that many researchers in design sciences are considering them. This new fact comes like a logical evolution from expert practice (in the sense of social sciences and in which little computerization is proven to be possible), towards an axiomatization as an inescapable precondition of a computerization.

But this statement can be argued since it is dissociating man from creative act by claiming that he might not be responsible of its control. However we undoubtedly know that this is not the case, the error comes according to us from the amalgam between creativity, creative process and human intrinsic aptitude [4]. If the process of implementation of the human creativity is observable, teachable, reproducible, the human actor at the heart of this process must develop particular aptitudes in problem solving [5].

\subsection{How a computer can support an inventive mode of design?}

This title is shocking in itself by the fact it partially associates two words "computer and invention". However as we described above, we do not fully associate invention and creativity. There is thus no question of bringing forward the assumption that creativity is computable, but simply to assert inventive steps assistance to the assistance of formulation and resolution of a certain typology of problems: inventive problems.

In our Design approach, our starting point concerning creativity is thus neither to supplant it, nor doping its processes of emergence, but to address the capacity of a designer to solve problems related with the specific situation he is facing with.

Regarding computerization of certain tasks related to designers, our choice was logically turned towards those in which a computerization would prove to be beneficial. In a past contribution, we proposed to assist extraction and storing of multi-disciplinary knowledge [6]. Later, we proposed to handle this knowledge and advanced specific representations useful for decisions in R\&D departments [7].

In short, among the essential functionalities that a computer can handle to assist a designer in logic of inventive design, we propose to treat the following ones:

- To assist expert questioning and to transpose his knowledge in components of inventive problems (reduced in our case to contradictions).

- To store this knowledge in templates allowing their re-uses, their dynamic iterations.

- To assist link constitution of these components to provoke all the possible interpretation based upon the provided representation. 


\subsection{Zooming on the formulation of inventive challenges}

The characteristic for a problem to be declared "inventive" was already clarified by Altschuller in TRIZ theory (1). Here, we will only use the ontology of a contradiction posed according to the fundamentals of the theory. The challenging aspect is observed when an unsolved situation possesses outcomes potentially carrying both substantial benefits for the company but also some risks.

Let us remind that a contradiction within the meaning of TRIZ (see also figure 1) can be modeled as follows:

An Active Parameter (APx) having two states of values diametrically opposed (Va and Vā) influencing respectively two evaluating parameters (EPn and EPm). The state of contradiction is obtained since the opposite state (Va $\rightarrow$ EPm and Vā $\rightarrow \mathrm{EPn})$ is also true.

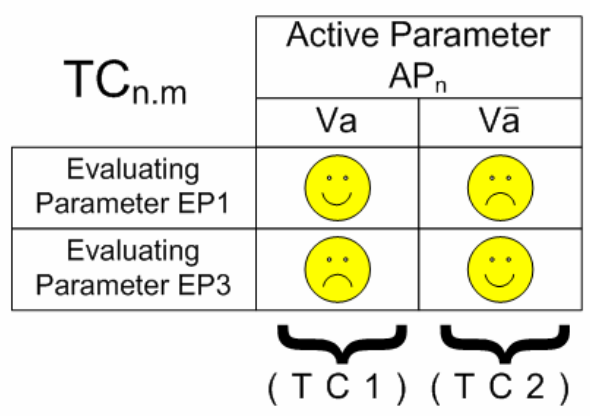

Figure 1 Schematic illustration on a contradiction

In TRIZ, we can associate an expressed physical contradiction to an inventive challenge when the two values of its AP are diametrically opposed and that the impossibility of placing AP at the same time in Va state AND in Vā state is ever more critical. Another element in the challenging value of a contradiction is the consequences of its solving; when above its own pair of EPs, its solving is diffused among many other EPs pairs (or other contradictions).

But such contradictions, when facing high challenges, are not easy to locate in the dense quantity of contradictions at the origin of complex problems. Our objective through the coming section is to propose a possible representation of multiple contradictions such as it emphasizes a reduced amount of contradictions at the heart of great challenges. 


\section{Supporting the selection of inventive challenges}

\subsection{On the acquisition of contradictions}

Firstly, we will consider the acquisition of contradictions and the way this fundamental stage of inventive problems solving can be conducted. The synthesis of a contradiction passes by specific processes allowing the emergence of parameters related with the studied system. Among these processes, let us note three principal stages:

- Description of a first set of parameters by the comprehension of past-present evolution dynamics of a given system. This process is primarily supported by multi-screen analysis [8].

- Description of a second set of parameters by studying the positioning of the system through laws of engineering systems evolution. Here the need is to confront the studied system with each law and analyze its evolution by characterizing it in terms of parameters [9].

- The first two processes having highlighted a group of contradictions, it is then appropriate to differentiate the Active Parameters from the Evaluating ones. Then, respectfully to the ontology of a contradiction, to associate APs and the EPs by means of their respective Values (Va and Vā). The incompleteness of these associations (according the described contradiction pattern) forces to identify the missing components. This constitutes also another mean of parameter's emergence.

The outcome of this last stage is that a more or less important set of contradictions will be disclosed. But the use of methods and tools extracted from TRIZ can be considered only on the basis of one of them. In the case of complex problems, it's a large amount of contradictions which will be synthesized; problems to insulate the contradiction to be engaged in a tool or a method of TRIZ for resolution still remain.

\subsection{On the concept of contradictions cloud}

Our past reports led us to understand that contradictions in complex situations are numerous and connected to multiple problems [10]. Thus, these contradictions, to be differentiated, must be associated to particular characteristics leading, when associated to values, to a graphical representation.

Our observations of these characteristics led us to highlight three elements of contradiction characterization. 


\section{First differentiation: Importance}

The contradictions components (an Active Parameter a pair of Evaluating Parameters) do not have the same importance; Evaluating Parameters are more characterizing the essentials of the problem than others. Thus, it is possible to associate a qualitative value to each Evaluating Parameters which compose a group of contradictions. This evaluation has only the aim to place them on a relative scale of importance. The essence to be preserved among parameters relationship is the scale amplitude to be simply established around a certain amount of divisions out of a scale allowing this differentiation.

Then, the role of the Active Parameter within a contradiction is not of the same order as the one provided by Evaluating Parameters. Very often, the active parameter represents the element on which it is necessary to act in a contradiction. We propose that the element which will allow differentiating a set of active parameters between them articulates around the potential impact that this APx (setted at Va AND Vā) will have on the problems to which it is related. In other terms an APx is going to atrophy or dope the importance of a pair of EP since we observed in Apx an evident capacity to influent EP's importance. The given coefficient $(\alpha)$ will then allow the addition of both EP's $(E P n+E P m)$ to be reduced when facing a weak impact or to be multiplied when facing a strong impact. For this multiplying coefficient, we agreed on a range from 0,5 to 2 .

Thus, the first criterion of differentiation between TCs will be following form:

$$
\begin{gathered}
\mathrm{X}=\alpha \mathrm{APx}(\text { Coef. EPn }+ \text { Coef. } \\
\text { EPm })
\end{gathered}
$$

Where:

$X$ : the association of an AP and its pair of opposite EP'S

$\alpha$ : the multiplying coefficient applied to the concerned AP

Coef. EPn and Coef. EPm: the two values of relative importance for each EP simply added.

\section{Second differentiation: Universality}

In our observations of the typology of a set of contradictions, we noted that EPs are qualifying the objectives sometimes hidden in inventive challenges. Some of these EPs seem to appear in a recurring way in a large amount of contradictions. This observation led us to build the assumption that a simple measurement of the universality of a contradiction could be established. This measurement would aim at specifying that a contradiction having EP recurrently present in a large amount of other contradictions represents the universality of this same contradiction. The universality criterion $\mathrm{Y}$ thus takes the following form:

$$
\mathrm{Y}=\mathrm{Q} \mathrm{EPn}+\mathrm{Q} \mathrm{EPm}
$$


Where:

$Y$ is the universality of a contradiction;

$Q$ EPn, $m$ : corresponds to the quantity of occurrence the EP has in a set of TC's.

\section{Third differentiation: Amplitude}

In the structure of a contradiction, an AP is associated with a pair of EPs. But in a more general way, the same AP is often associated with a variable quantity of EPs. As a result, some AP involve the opposition of only one pair of EPs whereas others have an impact on a large amount of EPs and involve the opposition of a consequent series of pairs of EPs. Our proposal is thus to establish a third criterion of differentiation $\mathrm{Z}$ related to contradictions which would specify their amplitude by the sum of EPs pairs each AP is attached to within a contradiction group. This criterion would take the following form:

Where:

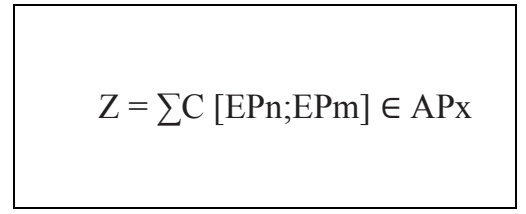

$Z$ is the criterion of amplitude;

$C$ represents any couple EPn; EPm

We thus have 3 criteria $\mathrm{X}, \mathrm{Y}$ and $\mathrm{Z}$ to differentiate contradictions. While placing criteria $\mathrm{X}$ and $\mathrm{Y}$ according to a system of axis, we obtain a cloud of dots. By associating each point of this cloud criterion $\mathrm{Z}$, we obtain (as represented on figure 2) a group of dots with variable diameters. 


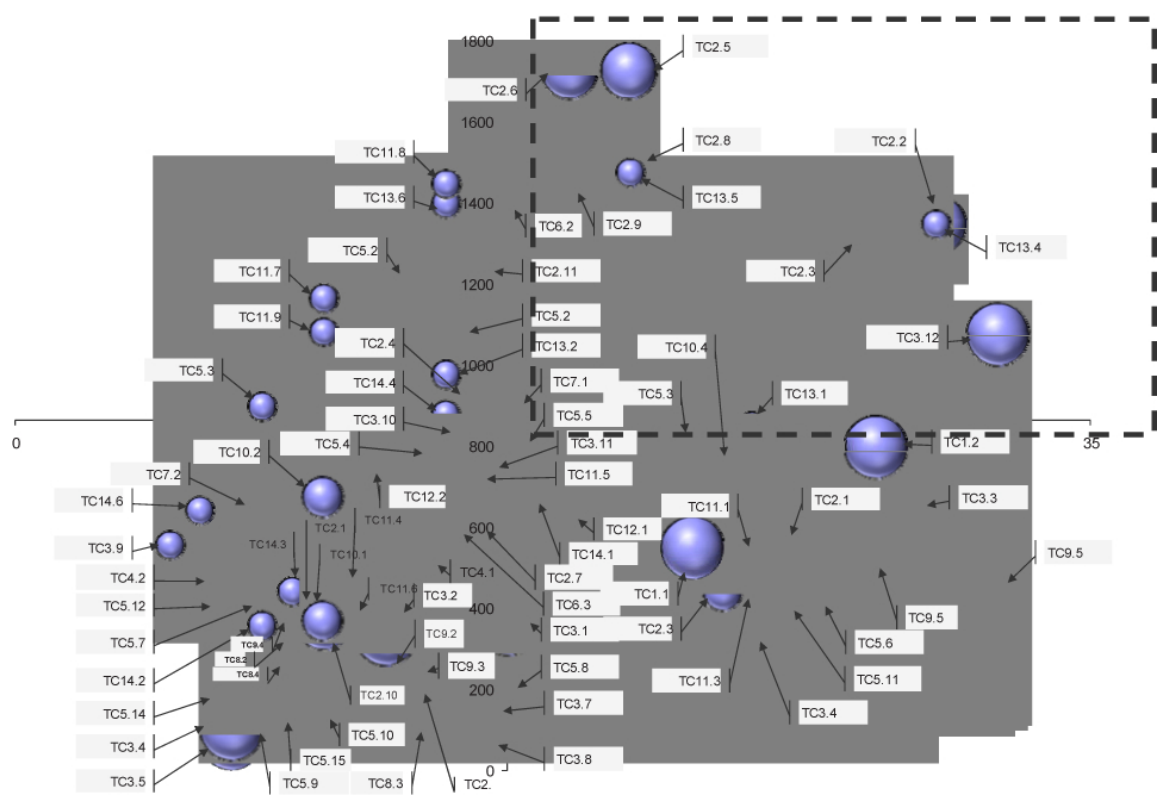

Figure 2 Cloud of contradictions with highligted top right corner

In such a representation, the zone of points in top right of the graph is of a particular importance. In order to highlight an even reduced amount of contradictions, a first method consists in isolating the set of contradictions of the top right quarter (figure 2).

But in the cases of complex situations, where contradictions are numerous, this pruning can not be enough. In such cases, we propose to highlight the top right front of points of this quarter cloud (figure 3).

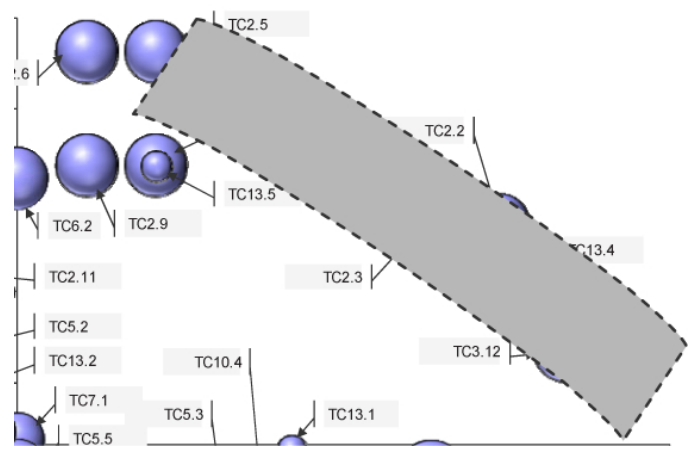

Figure 3 Dot's front zone of the top left quarter 
Let us return now to the objective of such a representation. We have an important set of contradictions representative of a set of expressed problems in a given situation. Our main objective is to put the designer in front of his inventive challenges, those impacting most consequently on the set of problems of its initial situation. The reduced dots on the upper right front thus represent a reduced amount of variants to address an inventive way complex problem. But assuming that the result of the analysis of such a graph is a set of decisions which will initiate a set of $R \& D$ activities, we are facing with a situation where it will be necessary to express the potential resources of R\&D actions. If for example a company has the capacity to address several R\&D axes in parallel, several choices on the upper left front face could be made. If the R\&D efforts must be reduced, a single choice (or a reduced amount of dots) will have to be selected.

\subsection{On the computerization of the process}

In a past contribution [11], we had already proposed a data-processing method for revealing contradictions materialized in a computer module. In order to associate this module the results of the analysis expressed in the preceding paragraph, we conceived a graphic extension of this module featuring the cloud of dots and the highlighting of its upper left front (figure 4).

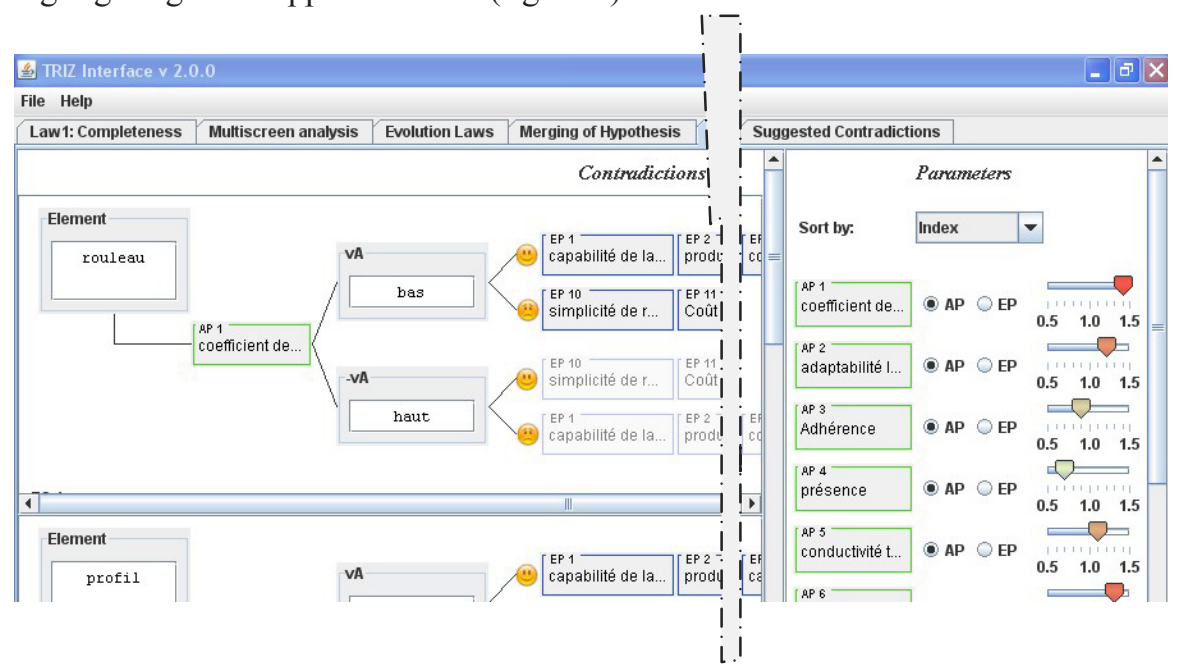

Figure 4 TRIZ acquisition screen capture - "Parameters \& Contradictions" menu. 


\subsection{Results of experimentation of the new TRIZ acquisition module in two industrial cases}

In the phase related to expert knowledge formalization of the industrialists we met, we tried out our module in two different industrial situations.

The first case was treated at a large automotive supplier, manufacturer of windshield wipers, the second in a steel industry in continuous annealing processing. The two cases treated summarized with their essential question was expressed to us the following way:

- Case 1: How to provoke the evolution of the windshield wiper from its present generation to its future?

- Case 2: How to decrease the amount of production stops in the manufacture of flat carbon steel sheets in continuous annealing processes?

Following the contradictions synthesis process in our two cases, we obtain the following results (figure 5):

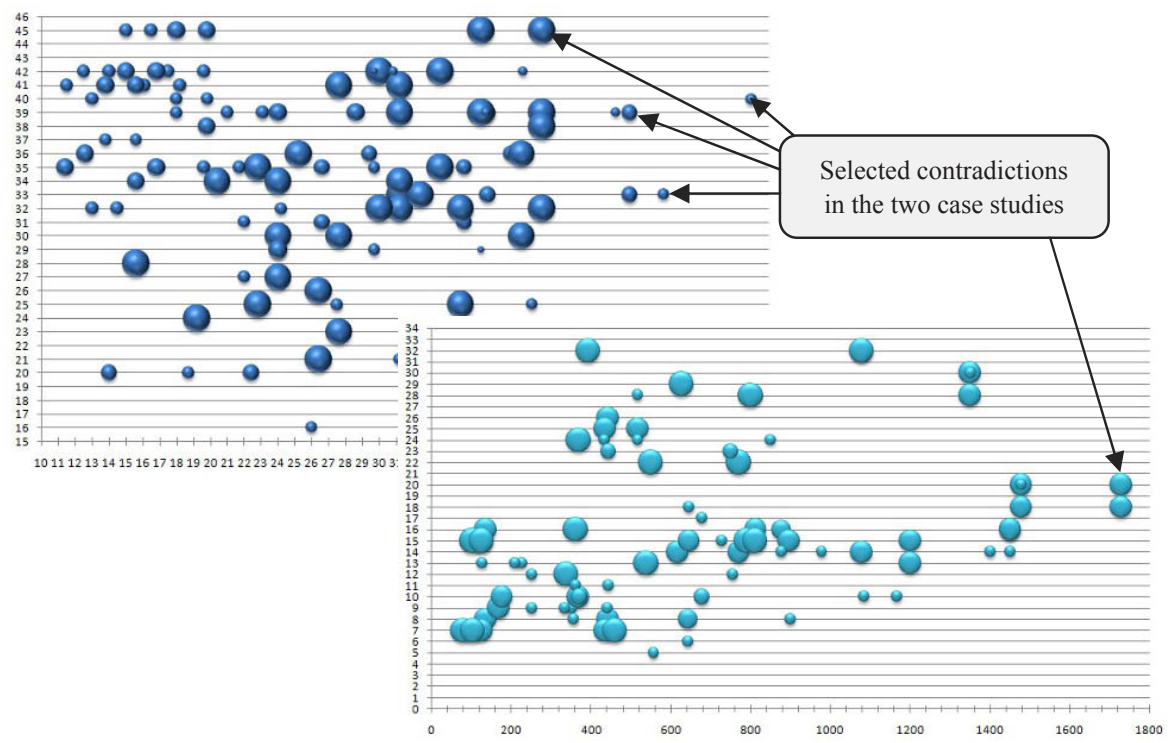

Figure 5a\&b Graphical results (Dots clouds) of the two case studies 


\subsection{Analyzes of the $R \& D$ decisions following the interpretation of the graphic data}

In Case 1, the R\&D decider agreed to engage the resolution of the TC3.12. This contradiction is placed on the left higher corner (see figure 5a). The current status of this case study is that several Solution Concepts have been disclosed out of the problem solving process and two of them are currently under patent applications. Another partial result is that an R\&D activity has been launched and consists in building a specific machine for measuring accurately the impact between several critical Evaluating Parameters since some of our assumptions during the meetings have found unprecise answers. In order to move further regarding this issue, it has been decided to engage a relation with a subcontractor to build the manufacturing means to perform these tesings and procesely characteraize the relation between these EPs.

In Case 2, the study group in agreement with the person in charge of the project, decided to engage the resolution of following contradictions: TC6.1 TC10.3 - TC2.8 - TC1.7 placed within and above the highlighted zone (see figure $5 b)$. After the solvinf part of our activities, eleven solution concepts have been disclosed and classifyed in six different categories. Three categories have been placed in R\&D activities priorities while one category has been sent to a subcontractor for calculations and characterization of the solution. The current status is that calucations came back with positive results, confirming what we felt during the sessions. The next step is to engage the realization of a prototype on a testing site for validation of calulation results.

\section{Discussions}

\subsection{On methodology related to the choice}

By considering as a starting point contradiction, let us remind that it is possible to implement the methods and tools brought by TRIZ the following way (figure 6).

We are then facing with a set of techniques which can be implemented to converge towards Solution Concepts recognized as inventive if they indeed solve at least the initial contradiction. Our objective is definitely not to obtain a large amount of concepts, but to increase the effectiveness of the problem solving step, it is thus important to choose only a reduced amount of contradictions, but to choose those which will increase the potential, when associated to their synthesized concepts, to significantly impact on the problem network we started with. 


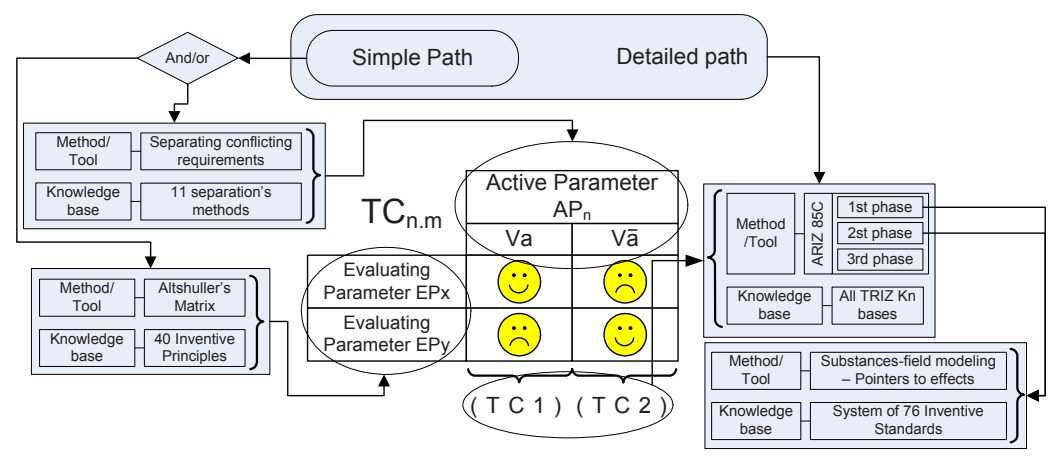

Figure 6 Addressing a contradiction with tools and methods brought from TRIZ

Another advantage of such a computerization is that it makes interactive and dynamics the graphic model established and the strategy of choice that the designer may start with is eased. Thus, if the set of contradictions (cloud of dots) on the graph is directly related to its values $\mathrm{X}, \mathrm{Y}$ and $\mathrm{Z}$, the designer can, for instance, decide to modify the parameter $\mathrm{Y}$ and to allocate a different ratio of importance to EPs since a particular strategy justifies it. It was the case in our study 1 where two parallel objectives were to be proposed to the decision makers. The first consisted in adopting a design of windshield wiper aiming at reducing the production cost without making compromise compared to the performances produced. In the same time, the R\&D of this company wished to compete with another equipment supplier on several markets (with car constructors) by offering a new product whose performances would be increased and would allow him to be placed over its direct competitors by the originality and the effectiveness of its solution. These two approaches required engaging the resolution of different contradictions. They were re-stated ahead by a quasi-instantaneous rebuilding of the cloud thanks to computerization. The draback of such a freedom is that parameters value may be adapted until an expected result is reached. It is therefore important not to engage a value modification based on an individual designer's desire but until a majority of team members commonly agreed on a modification based on quantitative physical realities (not on personal wishes).

In the case of flat steels, four contradictions were chosen by the group of study, some of them not being located on the upper left front. This choice can be explained by the decision of the group to address a wider ways of resolution of the set of problems settled at the beginning. The strategy was here to isolate a reduced amount of contradictions but resulting from different APs. Indeed, to address two or several contradictions from the same AP often invites us to concentrate during the solving phases on the same operational zone. The resultant is that the solution concepts sometimes emerge in a recurring way from one contradiction to the other. On the other hand, when the AP at the origin of a contradiction is different, we have a new angle of observation of the problem and the concepts which 
emerge give solutions of another nature. The objective of the second company being to eliminate a large amount of problems from the starting network, we concentrate our R\&D efforts in a larger set of directions so as to eliminate a maximum of problems as a result of this new strategy.

\subsection{On the universality of the process}

Another remark to be formulated relates to the fact these two companies present a significant organizational structure, and problems of a very different nature were expressed. The first one expressed a problem of product evolution, whereas the second expressed a set of problems involved in a manufacturing process. We thus observe that our procedure, associated the notion of the generic management of complex problems, is not significantly influenced by the nature of the addressed situation. The limits of such an assertion are related to the fact that the two cases presented an identical corpus of knowledge from the fields of mechanical engineering. However, it is already an emerging universality and our further work will aim to test it in distant fields than traditional engineering disciplines.

\subsection{On the positioning of the process regarding TRIZ body of knowledge}

From our investigations of TRIZ body of knowledge and the synthesis we made in the past, we remarked only rare contributions evoking the concept of management of multiple contradictions [12]. In spite of the fact that Altshuller himself evoked these problems, the convergence towards a relevant contradiction was only rarely addressed. In the software proposals where partial TRIZ elements can be found, some attempts at listing problems were made, but they do not rely on the axiomatic expression of a contradiction as recommended by one of the fundamental axioms of TRIZ. Some other work are currently expressed through the research around OTSM-TRIZ [13] but up to now a pragmatic way to support, operate and compute these problems is still not published in international peer reviewed journal or conference.

\section{Conclusions and further works}

It has been observed, through two industrial studies, that the process at the origin of this article contributes to the formalization of inventive practices in R\&D. Not only because it implements a pragmatic way to operate the fundamental of TRIZ, but also by the fact that such a computerization is likely to facilitate the adoption of new practices in companies. It remains however many axes in which further 
research is necessary. Among those, the formalization of organizational procedures to be integrated and adapted to companies typologies, customized to initiate an evolution of their design practices. Another one is the adaptation of Artificial intelligence techniques to automatically capture in texts, necessary knowledge to the gathering of contradictions in specific fields [14]. Our approach therefore has perspectives to evolve, compared to its first data-processing presented versions. We also note that it appears legitimate in the long term, to observe its output interfaced with the traditional CAD tools [15]. But there is still a consequent a gap to be filled prior to this. The one which separates an emitted inventive concept in a theoretical form and the necessary whole set of elements provoking its morphological characterization.

\section{References}

1. Coates J.F.: Technological Forecasting and Social Change, 64(2-3), pp. 121-32 (1996).

2. Chen Z.: Knowledge-Based Systems, 12(7), pp. 333-39 (1996).

3. Forgionne G., Newman J.: Decision Support Systems, 42(4), pp. 2126-36 (1996).

4. Bink M.L., Marsh, R.L.: Review of General Psychology, 4(1), pp. 59-78 (1996).

5. Treffinger D.J., Selby E.C., Isaksen S.G.: Understanding individual problem-solving style: A key to learning and applying creative problem solving, Learning and Individual Differences, in Press (2007).

6. Cavallucci D., Rousselot F.: Structuring knowledge use in Inventive Design, 2nd IFIP Working Conference on Computer Aided Innovation. Michigan, Springer, USA (2007).

7. Cavallucci D., Eltzer T.: Improving the Relevance of R\&D's Problem Solving Activities in Inventive Design Context, 16th International Conference on Engineering Design, ICED’07, Ecole Centrale Paris, Paris, France (2007).

8. Altshuller G.S.: Creativity as an axact science. The Theory of the Solution of Inventive Problems, Gordon and Breach Science Publishers (1984).

9. Salamatov Y.P.: System of the Laws of Technical Systems Evolution Chance to adventure, vol. 5, pp. 7-174, Karelia Publishing House, Petrozavodsk, (1991).

10. Cavallucci D., Khomenko N., Morel 15th International CIRP Design Seminar, p. 10, Shanghai, China (2005).

11. Zanni C., Rousselot F.: Towards the Formalization of Innovating Design: The TRIZ example, KES2006- 10th International Conference on Knowledge-Based \& Intelligent Information \& Engineering Systems, Bournemouth, UK (2006).

12. Pinyayev A.: Transition from the administrative contradiction to the technical one in the analysis of inventive problems (1990).

13. Khomenko N., Ashtiani M.: Classical TRIZ and OTSM as a scientific theoretical background for non-typical problem solving instruments, 7th ETRIA TRIZ Future Conference, Frankfurt, Germany (2007).

14. Tseng Y.-H., Lin C.J. Lin Y.I.: Information Processing \& Management, 43(5), pp. 1216-47 (1996).

15. Cavallucci D. Leon N.: Towards "inventiveness-oriented" CAI tools, WCC 2004 (IFIP 18th World Computer Congress), pp. 441-52, Kluwer Academic Publishers, Toulouse, France (2004). 